\section{Feasibility of day-case vitreoretinal surgery}

A Desai, A Rubinstein, A Reginald, M Parulekar and $\mathrm{V}$ Tanner

Conclusions These data suggest that many patients who are hospitalised overnight for VR surgery could be safely treated as day cases. Such a shift in the pattern of care for VR surgery could provide a significant improvement in health-care efficiency and minimise patient inconvenience.

Eye (2008) 22, 169-172; doi:10.1038/sj.eye.6702515; published online 21 July 2006

Keywords: day surgery; day care; ambulatory surgery vitrectomy; vitreoretinal

(50 retrospective and 50 prospective) undergoing VR surgery within the Royal Berkshire NHS trust were included. The retrospective arm aimed to identify the frequency and type of acute ophthalmic or medical intervention during postop overnight stay and the results were used to alter management in the prospective group. The prospective group consisted of patients undergoing a mixture of overnight stay and day-case surgery. All patients in the prospective group had routine subtenon marcaine anaesthesia together with prophylactic pre-operative intravenous acetazolamide. Patients deemed fit postoperatively were offered overnight ward discharge, with obligatory next-day review. Results In the retrospective arm, 56\% required oral nonsteroidal analgesia on the day of surgery and one patient required narcotic analgesia. Twenty-two per cent patients required intraocular pressure (IOP) control on the day of surgery and one patient required medical intervention in the form of urinary catheterisation. Nineteen patients required intervention on next-day review. In the prospective arm, $86 \%$ preferred day case and were suitable, $6 \%$ were suitable for day-case but preferred overnight stay and $8 \%$ were deemed not fit for discharge. No patient required narcotic analgesics. No patient discharged returned as a casualty overnight. Only one patient required topical beta-blocker for the control of IOP on next-day review.

\section{Introduction}

The majority of ophthalmic surgical procedures are now performed as day-case surgery. ${ }^{1-4}$ Patients requiring vitreoretinal (VR) surgery have traditionally been operated under general anaesthesia and admitted for an overnight stay in hospital following surgery. This was mainly due to ocular and systemic morbidity associated with VR procedures: ocular pain, raised intraocular pressure (IOP), and diabetes-related medical complications being the most important. $^{5-8}$ There has been a significant shift towards local anaesthesia for VR surgery, but there are no recent published data for day-case surgery. Previously, cataract surgery involved similar routine overnight stay. Today, day-case cataract surgery is recognised as a safe cost-effective alternative to overnight hospitalisation. ${ }^{9-10,12}$ This study examines the feasibility of provision of day-case VR surgery.

Day-case surgery is preferable because patients receive care that is better suited to their costs are also lower with greater opportunity for increased throughput and more efficient use of resources. The NHS Plan envisages that $75 \%$ of all elective surgery will be carried out a day case on basis in the near future. ${ }^{1,11}$

Patients and methods

One hundred consecutive VR procedures performed from November 2003 until wishes with less time spent in hospital. Hospital
Royal Berkshire and Battle NHS trust, Prince Charles Eye Unit, Reading and King Edward VII Hospital, Windsor, UK

Correspondence: V Tanner, Royal Berkshire NHS Trust, Prince Charles Eye Unit, Reading and King Edward VII Hospital,

Windsor SL43DP, UK Tel: + 4401753860441

E-mail: tannerone@aol.com

Received: 19 January 2006 Accepted: 6 June 2006 Published online: 21 July 2006 
September 2004 at The Royal Berkshire and Battle Hospitals were included in the study. Fifty case notes were retrospectively reviewed and 50 consecutive prospective patients were recruited into the study. In the retrospective group, the data collected included: patient demographics, past medical and ocular history, indication for surgery, surgical procedure, type of admission, type of anaesthesia, duration of procedure, supplemental postoperative analgesia, IOP lowering agents and the need for any medical intervention. Exclusion criteria included trauma cases and relatively minor cases already carried out on a day-case basis (eg, indirect laser, retinal cryopexy, removal of silicone oil, and pneumatic retinopexy).

In the prospective group, all patients were offered day surgery with obligatory next-day review or overnight hospitalisation. All patients underwent surgery in the morning session and were reviewed on the same day at 1700 hours, at day 1 and 2 weeks postoperatively. All patients received supplemental anaesthesia pre-operative with $3 \mathrm{ml}$ of marcaine $0.5 \%$ in the subtenon space, regardless of whether they had general or local anaesthesic. All prospective group patients also received $500 \mathrm{mg}$ of intravenous acetazolamide preoperatively. Additional data collected for this group included postoperative pain, as measured on the visual analogue scale.

Patients who requested overnight hospitalisation were asked if they would have preferred day surgery if next-day review were not mandatory.

\section{Results}

\section{Retrospective group}

The relevant patient data are presented in Tables 1 and 2 . The average age of the patients in the retrospective group was 59.5 years (range 25-96 years). Fifty-four per cent were male patients. Only eight patients $(16 \%)$ were admitted as day cases and 42 patients $(84 \%)$ stayed in hospital overnight. Eleven patients (22\%) had local anaesthesia and 39 patients $(78 \%)$ had general anaesthesia. The indications for surgery included: rhegmatogenous retinal detachment (25), retained lens fragments (four), diabetic vitreous haemorrhage (five),

Table 1 Patient data

\begin{tabular}{lll}
\hline & Retrospective data & Prospective data \\
\hline $\begin{array}{l}\text { Patient demographics } \\
\text { Male: female }\end{array}$ & $1.27: 1$ & $1: 1$ \\
$\begin{array}{l}\text { Age (years) } \\
\text { Day case }\end{array}$ & Range: $25-98$; average: 59.7 & Range: $19-88$; average: 60.5 \\
Type of anaesthesia & GA/LA: $39: 11$ & $32(64 \%)$ \\
Indication for surgery & Retinal detachment: 25 & GA/LA: $26: 24$ \\
& Diabetic vitreous haemorrhage: 5 & Retinal detachment: 21 \\
& Vitreous haemorrhage other causes: 4 & Diabetic vitreous haemorrhage: 8 \\
& Idiopathic macular hole: 7 & Vitreous haemorrhage other causes: 2 \\
& Epiretinal membranes: 4 & Macular hole: 4 \\
& Retained lens fragments: 4 & Epiretinal membrane: 8 \\
& Fungal endophthalmitis: 1 & Retained lens fragments: 6 \\
& Cryotherapy with scleral buckles: 10 & Floaters: 1 \\
& Vitrectomy with gas: 21 & Cryotherapy with scleral buckles: 5 \\
Type of procedure & Vitrectomy with oil: 9 & Vitrectomy with gas: 18 \\
& Vitrectomy without endotamponade: 10 & Vitrectomy with oil: 7 \\
& & Vitrectomy without endotamponade: 20 \\
& & Combined vitrectomy with oil and encirclement: 1 \\
& &
\end{tabular}

Table 2 Postoperative intervention in the retrospective group

\begin{tabular}{lll}
\hline & On the day of surgery & Next-day review \\
\hline Non-narcotic analgesics & 27 & 4 \\
Morphine & 1 & 0 \\
IOP control & 11 patients were given oral & 12 patients required IOP control (nine had \\
& acetazolamide & IOP $>30 \mathrm{mmHg}$ ) \\
Medical intervention & Urinary catheterisation & Transfer to surgical ward \\
Others & Nil & Choroidal detachment 1 \\
& & Displaced IOL 1
\end{tabular}


Table 3 Postoperative intervention in the prospective group

\begin{tabular}{lcc}
\hline & $\begin{array}{c}\text { On the day } \\
\text { of surgery }\end{array}$ & $\begin{array}{c}\text { Next-day } \\
\text { review }\end{array}$ \\
\hline Non-narcotic analgesics & $14(28 \%)$ & 2 \\
Morphine & 0 & 0 \\
IOP control & $12(24 \%)$ & 1 \\
IOP $>30 \mathrm{mmHg}$ & 4 & 0 \\
$\begin{array}{l}\text { Medical intervention } \\
\text { Pain } \geq 5 \text { as measured on the } \\
\text { visual analogue scale }\end{array}$ & $7(14 \%)$ & $\mathrm{Nil}$ \\
\hline
\end{tabular}

idiopathic macular holes (seven), macular pucker (four), non-diabetic vitreous haemorrhage (four), and one case with fungal endophthalmitis. The operative procedures are listed in Table 1.

During the afternoon following morning surgery, nonnarcotic analgesia in the form of oral codeine and paracetamol was administered to 28 patients $(56 \%)$, and one patient required morphine. Seven out of $10(70 \%)$ scleral buckling procedures required analgesia, compared to $16 / 30$ (53\%) for pars plana vitrectomy with endotamponade, and 5/10 (50\%) for vitrectomy alone. Oral acetazolamide (Diamox) was required in 11 patients (22\%) on the day of the operation to control IOP. One patient who underwent general anaesthesia had acute urinary retention and required catheterisation.

Next-day intervention was necessary in 19 patients. Raised IOP was documented in 12 patients and treated with topical IOP lowering agents and/or oral diamox. An IOP greater than $30 \mathrm{mmHg}$ was found in nine patients. Four patients required nonnarcotic analgesia for pain. On the next-day review, one patient was noted to have choroidal detachment, another had a malpositioned intraocular lens, and one patient was transferred to the surgical ward following urinary catheterisation (Table 2).

\section{Prospective group}

The relevant patient data are presented in Tables 1 and 3 . The average age of the patients in this group was 60.5 years (range 19-88 years) with equal numbers of male and female patients. Sixty-four per cent of patients were managed as day cases. At the 2-week review, a further $28 \%$ would have preferred day-case surgery, but chose not to because of mandatory next-day follow-up. Three patients preferred in patient care because of social reasons. Four patients were deemed not fit for same-day discharge. These were: one patient with severe learning disabilities related to Down's syndrome, one patient on renal dialysis, and two with poor vision in the fellow eye. Fifty-two per cent of patients underwent general anaesthesia compared to $48 \%$ who had local infiltration.
The indications for surgery and type of procedure are summarised in Table 1.

Nonnarcotic analgesia was requested by 14 patients, and none required morphine. Pain as measured on the 11-point (0-10) visual analogue scale was $\leq 4$ in $86 \%$ of patients. Seven patients had a score $\geq 5$, out of which three patients had a scleral buckling procedure.

Eight patients had IOPs between 21 and $30 \mathrm{mmHg}$ on the afternoon following surgery, which was treated with topical agents. The IOP was found to be $>30 \mathrm{mmHg}$ in four patients on the same day of surgery, with the highest recording of $36 \mathrm{mmHg}$. These patients required oral acetazolamide in addition to topical agents. No patient had an IOP $>30 \mathrm{mmHg}$ on the day following surgery.

Three patients required intervention on the following day: two requested additional oral pain relief and one had topical beta-blocker to control IOP.

\section{Discussion}

Over the past decade, there has been a significant transition from in patient care to day-case surgeries notably in the case of cataract and squint surgery. ${ }^{1}$ We found no published data on day-case VR surgery in the past 15 years. Previous published results supported in-patient care because of significant ocular and systemic morbidity following VR procedures. . $^{5,13-17}$

In 1988, Isernhagen et al prospectively analysed 200 patients and concluded that in patient care was better suited for $52 \%$ of their patients with $40 \%$ requiring intramuscular injections for relief of pain or nausea and $15 \%$ requiring physician attention for systemic illness more than $5 \mathrm{~h}$ following surgery. In their survey, $79 \%$ patients indicated that they would be uncomfortable with ambulatory surgery. ${ }^{6}$ Ninety per cent of the patients had local anaesthesia with sedation. However, more recent published data show strong evidence of the effectiveness of local anaesthesia in intraoperative comfort and in controlling postoperative pain. ${ }^{7,9,18-20}$ Isernhagen's results were also skewed with $58 \%$ of eyes having previous retinal surgery.

In 2001, Fekrat et al reported on a series of 185 cases with retrobulbar analgesia with sedation. Forty-eight per cent required postoperative analgesia, with half of these requiring narcotic agents. This was more frequent in procedures that lasted more than $2 \mathrm{~h} .{ }^{13}$ In our series, none of the procedures lasted more than $2 \mathrm{~h}$ nor did any case under local anaesthesia require sedation. There are several other reports that support our findings of reduced pain and patient satisfaction for local anaesthesia. ${ }^{7,9,18-20}$

In September 2004, the in patient facility was withdrawn from the dedicated eye unit at King Edward 
VII Hospital, Windsor. This study was designed to identify the intervention necessary during in-patient stay and the feasibility of providing day-case surgery. Retrospective analysis of the intervention required during in patient care revealed mainly oral nonnarcotic analgesics and either topical or oral IOP lowering agents. As expected, pain is higher in patients undergoing scleral buckling procedures; however, this was adequately controlled with nonnarcotic analgesics. In the prospective arm, patients were encouraged to have local anaesthesia and all patients received $500 \mathrm{mg}$ of intravenous acetazolamide along with an additional $3 \mathrm{ml}$ subtenon infiltration of $0.5 \%$ marcaine to decrease the incidence of postoperative pain and IOP spikes. Our practice changed so that all IOPs were checked on the day of surgery. This allowed us to confirm that those eyes with high IOP were identified and adequately treated on the day of surgery. These measures proved to be highly effective and decreased the requirement for any analgesia at all from $56 \%$ in the retrospective group to $28 \%$ in the prospective group and for postoperative IOP control requiring oral acetazolamide from 22 to $6 \%$.

In the prospective group, at next-day review, $96 \%$ patients were comfortable and requested no additional analgesics and only one required topical IOP lowering agent for an IOP of $28 \mathrm{mmHg}$. The patient requiring IOP treatment actually had a pressure over $30 \mathrm{mmHg}$ on the day of surgery and should have been treated at this point. If this latter patient is excluded, then no patients required intervention on the day following surgery other than for oral analgesia.

Only $6 \%$ patients preferred in patient care and an additional $8 \%$ were deemed not fit for day-case surgery. The reasons for in patient surgery were mainly ocular and systemic comorbidity along with social factors.

We now routinely check IOPs at 1700 hours on the day of surgery and this has replaced the day 1 examination. We have successfully transitioned to day-case surgery without compromising patient safety.

The study is a longitudinal, observational study of 100 consecutive patients undergoing VR procedures. We did not match the retrospective groups with the prospective groups either in terms of indication for surgery or type of procedure. Our aim was to ascertain medical intervention or any adverse event occurring in the immediate postoperative period.

We conclude that day-case surgery is a feasible option in the majority of local and general anaesthetic cases.

\section{References}

1 Audit Commission (2001). Day surgery. Acute Hospital Portfolio Review of National Findings No. 4.

2 Beatty S, Kheterpal S, Eagling EM, O'Neill EC. Day-case trabeculectomies: safety and efficacy. Acta Ophthalmol Scand 1996; 74(2): 132-134.

3 Aquavella JV, John T, Garcia E, Rao GN. Outpatient keratoplasty. Am J Ophthalmol 1985; 100(2): 276-280.

4 Dresner SC, Klussman KG, Meyer DR, Linberg JV. Outpatient dacryocystorhinostomy. Ophthalmic Surg 1991; 22(4): 222-224.

5 Newsom R, Luff A, Wainwright C, Canning C. UK survey of attitudes to local anaesthesia for vitreoretinal surgery. Eye 2001; 15(Part 6): 708-711.

6 Isernhagen RD, Michels RG, Glaser BM, de Bustros S, Enger C. Hospitalization requirements after vitreoretinal surgery. Arch Ophthalmol 1988; 106(6): 767-770.

7 Rao GP, Wong D, Groenewald C, McGalliard JN, Jones A, Ridges PJ. Local anaesthesia for vitreoretinal surgery: a case-control study of 200 cases. Eye 1998; 12(Part 3a): 407-411.

8 Wilson D, Barr CC. Outpatient and abbreviated hospitalization for vitreoretinal surgery. Ophthalmic Surg 1990; 21(2): 119-122.

9 Hodgkins Pr, Luff AJ, Morrel AJ, Botchway LT, Featherston TJ, Fielder AR. Current practice of cataract extraction and anaesthesia. Br J Ophthalmol 1992; 76(6): 323-326.

10 Eke T, Thompson JR. The National Survey of Local Anaesthesia for Ocular Surgery. II. Safety profiles of local anaesthesia techniques. Eye 1999; 13(Part 2): 196-204.

11 Day surgery operational guide. Waiting, Booking and Choice. Department of health; 2002.

12 Cresswell PA, Allen ED, Tomkinson J, Chapman FM, Pickering S, Donaldson LJ. Cost effectiveness of a single-function treatment center for cataract surgery. J Cataract Refract Surg 1996; 22(7): 940-946.

13 Fekrat S, Elsing SH, Raja SC, Campochiaro PA, de Juan Jr E, Haller JA. Eye pain after vitreoretinal surgery: a prospective study of 185 patients. Retina 2001; 21(6): 627-632.

$14 \mathrm{Wu}$ WC, Schachat AP. Transfer from ophthalmology to another service is a marker of high risk medical events. Ophthalmic Surg 1991; 22(1): 7-11.

15 Cannon CS, Gross JG, Abramson I, Mazzei WJ, Freeman WR. Evaluation of outpatient experience with vitreoretinal surgery. Br J Ophthalmol 1992; 76(2): 68-71.

16 Leaver P. Prospects for day-case vitreoretinal surgery. Br J Ophthalmol 1992; 76(2): 65.

17 Fleischman JA, Lerner BC. To admit or not to admit, that is the question. Arch Ophthalmol 1988; 106(11): 1501.

18 Newsom RS, Wainwright AC, Canning CR. Local anaesthesia for 1221 vitreoretinal procedures. $\mathrm{Br} \mathrm{J}$ Ophthalmol 2001; 85(2): 225-227.

19 Calenda E, Olle P, Muraine M, Brasseur G. Peribulbar anesthesia and sub-Tenon injection for vitreoretinal surgery: 300 cases. Acta Ophthalmol Scand 2000; 78(2): 196-199.

20 Knight HM, Newsom RB, Canning CR, Luff AJ, Wainwright AC. Local anaesthesia for vitreoretinal surgery: an audit of patient and surgical experience. Eur J Ophthalmol 2001; 11(4): 366-371. 